R E V I S T A

de la

C E P A L

NUMERO 58

ABRIL 1996

SANTIAGO DE CHILE

GERT ROSENTHAL

Director

EUGENIO LAHERA

Secretario Técnico

NACIONES UN I DAS 
Aníbal Pinto Santa Cruz

Gert Rosenthal

Los paradigmas de la política social en América Latina

9

Rolando Franco

Virtudes y limitaciones de los mapas censales de carencias críticas

Rubén Kaztman

Centroamérica: inflación y estabilización en la crisis y poscrisis

Hubert Escaith, Claudia Schatan

El Estado, la empresa y la restauración del paradigma neoclásico

A. Barros de Castro

Globalización y pérdida de autonomía de las autoridades fiscales, bancarias y monetarias

Juan Carlos Lerda

El contexto macroeconómico y la inversión: América Latina

a partir de 1980

Graciela Moguillansky

Los derechos de propiedad y el mercado de la tierra rural en América Latina

Frank Vogelgesang

México: Plan y coyuntura

David Ibarra

Comercio exterior y medio ambiente: experiencias en tres sectores exportadores chilenos

Imme Scholz

El desafío competitivo para la industria brasileña

João Carlos Ferraz, David Kupfer, Lia Hagenauer

Indicadores de la política fiscal: diseño y aplicaciones para Chile

Ricardo Mariner

La CEPAL y la sociología del desarrollo 


\section{Comercio exterior y medio ambiente: experiencias en tres sectores exportadores chilenos}

\section{Imme Scholz}

Investigadora,

Instituto Alemán de Desarrollo, Berlín
Este artículo presenta los resultados de una investigación sobre la capacidad de ajuste ambiental de empresas exportadoras chilenas en varios sectores: madera, celulosa, industria del mueble y cultivo de salmón, ostras y ostiones. El estudio tuvo como punto de partida las exigencias ambientales de Alemania y de Europa en general respecto de productos y procesos, las que ejercen una presión de ajuste sobre éstos. Luego de pasar revista al entorno ofrecido por Chile para ese proceso de adaptación (sección I), se examinan las exigencias señaladas y su status dentro del régimen multilateral del GATT (sección II). En seguida se especifican los requerimientos que las reformas ambientales plantean a los agentes económicos, políticos y sociales en materia de gestión y ajuste en los diferentes niveles en que ellos actúan; estas reflexiones constituyen el marco analítico de la investigación, en tanto que el planteo del problema invita a aprovechar el concepto de la competitividad sistêmica en calidad de marco heurístico para definir con más precisión las exigencias impuestas a los agentes que operan a nivel macro, meso y micro (sección III). A continuación se profundiza más en la capacidad de ajuste ambiental de las empresas pertenecientes a los sectores estudiados (sección IV), y se presentan por último (sección V) las conclusiones del trabajo. En las empresas (nivel micro), la orientación hacia estándares de competitividad internacionales fomenta importantes procesos de aprendizaje e innovación tecnológica y organizativa, mientras que a nivel meso y macro (entorno empresarial y actividad reguladora estatal) existen deficiencias que limitan la capacidad de ajuste de las empresas, obstaculizando de esta manera el aprovechamiento económico eficiente de ventajas comparativas dinámicas ligadas al manejo sustentable de los recursos naturales chilenos. 


\section{I}

\section{Introducción}

Las empresas de los países en desarrollo orientadas a la exportación vienen encarando cada vez más exigencias ambientales en relación con sus productos y sus métodos de producción (Scholz, 1993; Wiemann y otros, 1994). Estas nuevas exigencias empujan a las empresas a emprender complejos procesos de aprendizaje en materia de gestión ambiental, tecnología y organización, procesos que pueden requerir ajustes simples o muy complicados de las líneas de productos $\mathrm{y}$ de las fases del proceso productivo. A juzgar por las experiencias de los países de la Organización de Cooperación y Desarrollo Económicos (OCDE), el éxito de esos procesos de aprendizaje depende no sólo de la presencia de empresas innovadoras, sino también de un contexto macroeconómico, político, jurídico y administrativo que genere incentivos para compatibilizar cada vez más la producción y los productos con la protección del medio ambiente y, además, de un entorno empresarial cuyas instituciones (de capacitación y perfeccionamiento, investigación y tecnología, financiamiento y política comercial y ambiental) apoyen la búsqueda de soluciones adecuadas.

Chile es uno de los países más indicados para analizar los procesos de adaptación ambiental vistos en este marco conceptual. Cuatro son las razones para ello:

i) El modelo económico que, con su clara orientación hacia la competitividad y el mercado mundial vino a reemplazar en 1973 a la estrategia de la sustitución de importaciones, ha dado pie al surgimiento de un sector exportador potente y dinámico que hoy día genera alrededor de la cuarta parte del PIB. ${ }^{1}$ En las áreas claves del sector exportador, el régimen de libre comercio ha hecho surgir un sector empresarial entrenado para sondear los requerimientos del mercado mundial con respecto a productos y procesos y capa-

D La investigación en la que se basa este artículo fue efectuada por un grupo de trabajo del Instituto Alemán de Desarrollo, de Berlín, en el que participaron, además de la autora, Karola Block, Karen Feil, Martin Krause, Karolin Nakonz y Christoph Oberle. El citado grupo recibió un apoyo considerable de ProChile, la agencia estatal de fomento a la exportación. Para un informe exhaustivo sobre la investigación y sus resultados en cada uno de los sectores estudiados, véase Scholz, Block, Feil, Krause, Nakonz y Oberle (1994). La autora agradece la traducción del artículo a Carlos Átala.

${ }^{1}$ En 1993, el PIB ascendió a 43700 millones de dólares y la exportación total a 9400 millones (bfai, 1995). citado para adaptar la organización de sus empresas a esos requerimientos.

ii) Buena parte de la competitividad internacional de las empresas chilenas de exportación sigue dependiendo de la explotación de ventajas de localización estáticas, es decir, de los bajos costos del factor trabajo, de condiciones climáticas favorables y de la abundancia de recursos naturales. Pero la fase de explotación extensiva de esa fuente de competitividad empieza a tropezar con sus propios límites. Uno de ellos es la demanda de mejoras salariales para las mayorías, demanda que viene en aumento desde el retorno de la democracia en 1990 y cuya satisfacción parece factible dado el sostenido crecimiento económico del país. El otro límite está en los costos ambientales ligados al éxito de la nueva estrategia de desarrollo: el crecimiento acelerado y el desarrollo dinámico de los nuevos sectores exportadores basados en recursos naturales - ante todo el pesquero, el forestal y el hortofrutícola - entrañan el peligro de agotar determinados recursos naturales, además de contaminar en alto grado el aire, las aguas y el suelo. ${ }^{2}$

iii) Una política económica de cara al futuro debe apuntar a dos cosas: primero, asegurar a largo plazo la competitividad internacional de la economía chilena, extendiendo las proyecciones sociales del crecimiento, y segundo, disminuir el impacto ambiental de los patrones de producción. A tal efecto será preciso aumentar la creación de valor agregado, elevar la productividad y desarrollar sectores más complejos y menos dependientes de los recursos naturales. Si se atiene a los dos propósitos señalados, la industrialización orientada hacia el exterior y apoyada en los sectores exportadores dinámicos que se basan en las

\footnotetext{
${ }^{2}$ Conforme a la distribución regional de los nuevos sectores exportadores, todos los problemas ambientales -salvo la sobrepesca de las aguas litorales- acusan una acentuada dimensión regional: la degradación y la tala rasa de los bosques nativos, que pone en peligro la biodiversidad, se concentra en el sur del país; la contaminación del suelo y de los recursos hídricos a causa del monocultivo se produce ante todo en las regiones especializadas en la silvicultura, la horticultura o la fruticultura; en Santiago y otros conglomerados urbanos, la contaminación aérea, el tráfico, el transporte y la eliminación de residuos son fuentes de grandes dificultades (Sanhueza, 1992). Sobre la distribución regional de los nuevos sectores económicos y sus impactos ambientales, véase Dockendorff y otros (1990), citado en Maggi (1994).
} 
materias primas (la denominada "segunda fase" del modelo exportador $)^{3}$ podría abrir márgenes de maniobra para aumentos salariales e impedir que Chile se desenganche del desarrollo tecnológico. Las posibilidades de reformar la economía chilena en favor del medio ambiente residen en los efectos sinergéticos que puedan derivar de vincular las medidas promotoras de la productividad y la eficiencia con la protección del medio ambiente y los recursos naturales, a nivel tanto empresarial como sectorial. ${ }^{4}$

iv) Desde 1990, año en que dejó de existir el régimen militar, han venido surgiendo los primeros elementos de una regulación ambiental a nivel del Estado $;^{5}$ el debate público sobre el particular permite inferir una concientización relativamente amplia respecto del medio ambiente. Pero las reformas se ven al mismo tiempo obstaculizadas por tres factores característicos en los países en desarrollo que han abandonado la estrategia de sustitución de importaciones con su alto grado de intervención estatal. En primer lugar, la ideología neoliberal predominante hace que las intervenciones del Estado parezcan ineficientes en com- paración con las regulaciones de mercado y lleva a desconocer las funciones positivas de la gestión pública. Sucede, sin embargo, que la acción del Estado no se limita a establecer y consolidar el contexto institucional propicio al libre despliegue de los individuos y de los subsistemas integrantes de una sociedad, sino que, entre otras cosas, coordina los intereses diferentes o divergentes en aras del bien común y da coherencia a las distintas políticas sectoriales. En segundo: lugar, la reducción del aparato del Estado ha provocado la pérdida de capacidades de gestión, planificación y fiscalización. En tercer lugar, se ha acrecentado con esto la influencia de los grupos de interés poderosos a la hora de formular, ejecutar o bloquear las iniciativas parlamentarias, de modo que las soluciones a los pro $^{\wedge}$ blemas ambientales se ven entorpecidas por intereses económicos particulares. ${ }^{6}$

En Chile, por lo tanto, hay necesidad de reformas a nivel empresarial, sectorial y político-adminis ${ }^{\wedge}$ trativo, todo ello dentro de un contexto favorable en términos políticos y económicos, a pesar de las limitaciones mencionadas.

II

\section{Exigencias ambientales a productos y procesos}

\footnotetext{
${ }^{3}$ Véase Madrid y Ominami (1989) y Mármora y Messner (1992), también Messner (1993) sobre el desarrollo de potenciales de competitividad en el sector exportador maderero chileno.

${ }^{4}$ Un uso más eficiente de las materias primas y la energía ayuda a reducir los costos de las empresas y permite aprovechar los recursos con más cuidado, facilitando entre otras cosas su regeneración y reduciendo el consumo energético y las emisiones consiguientes. Lo que continúa sin respuesta es el problema de limitar el consumo absoluto de recursos naturales y ambientales que acompaña a la industrialización y a la difusión de los patrones occidentales de consumo en los países en desarrollo (suficiencia en contraste con eficiencia).

${ }^{5}$ En 1990 se fundó la Comisión Nacional del Medio Ambiente (CONAMA) a cuyo cargo están la coordinación y la concepción de la política ambiental; el desarrollo, la ejecución y la fiscalización de la política ambiental a nivel sectorial sigue siendo competencia de los respectivos ministerios. La primera ley ambiental sectorial fue la Ley de Pesca, aprobada en 1991, que implantó un régimen de cuotas para la explotación y reproducción de los recursos pesqueros. En 1994 fue aprobada la Ley sobre Bases Generales del Medio Ambiente, que sirve de fundamento a otras leyes, decretos y normativas protectoras aún pendientes, regulando además la implantación de instrumentos tales como la evaluación del impacto ambiental, la responsabilidad legal por daños ambientales, y los impuestos, contribuciones y licencias. Las leyes y otros recursos legales se guían por el principio de la prevención y del que contamina paga (Chile, 1994). En CPMA (1992) se ofrece una idea general del debate chileno en torno a los modelos reguladores, instrumentos y campos de acción que comprende la política ambiental.
}

Las exigencias ambientales relativas a los productos vienen cobrando cada vez mayor importancia en Alemania y en otros países europeos. Tres son las razones que explican este fenómeno: primero, porque lo\$ consumidores, al estar mejor informados que antes sobre las propiedades contaminantes o nocivas de un bien, se guían por criterios ambientales a la hora de adquirirlo. Segundo, porque esa actitud más vigilante del consumidor incrementa la presión sobre el poder legislativo para que éste conjure los peligros potenciales que entrañan las sustancias tóxicas para el medio ambiente y la salud humana. Y tercero, porque la in-

\footnotetext{
${ }^{6}$ Gómez-Lobo y Jiles (1993, pp. 352 - 357) describen las negociaciones sobre la Ley de Pesca y analizan la poderosa influencia que ejercieron las grandes empresas pesqueras en favor de constituir consejos regionales que fijen el régimen de capturas en lugar de que lo hagan las instancias oficiales de ese sector. Esos consejos constituyen el instrumento a través del cual las empresas pueden impedir la creación de un sistema de cuotas individuales comerciables capaz de regular la extracción de recursos pesqueros y limitan mucho la libertad de acción de las instancias oficiales en pro de un manejo efectivo de esos recursos.
} 
dustria y el comercio, que han detectado ese cambio de actitud en el consumidor, están readaptando progresivamente su producción y la compra de sus insumos. Estas medidas de ajuste difieren mucho en sus alcances y van desde sólo aprovechar las oportunidades de comercialización ambiental hasta registrar los daños e impactos ambientales debidos a la producción para finalmente eliminarlos. Esta última opción es la que han elegido las empresas pioneras que han reconocido las ventajas competitivas de un acentuado perfil ambientalista y quieren explotarlas.

Las exigencias ambientales tocantes al producto toman diferentes formas. ${ }^{7}$ Los requerimientos legales obligatorios en forma de valores límites, normativas de uso o, según el caso, prohibiciones sobre sustancias peligrosas, actúan en defensa del consumidor, de la salud y de la seguridad laboral y, en el caso de los venenos ambientales, sirven para proteger además el aire, el suelo y el agua.

Paralelamente se vienen aplicando cada vez más instrumentos opcionales capaces de incentivar a las empresas para que elaboren productos de menor impacto ambiental. Figuran entre ellos la normalización industrial, que es concertada entre fabricantes y usuarios según criterios técnicos y que ya empieza a incorporar elementos ambientales. Otros instrumentos de aplicación voluntaria son el marcado de productos con un sello ecológico (OCDE, 1991; Jha, Vossenaar y Zarrilli, 1993) y los compromisos voluntarios contraídos por la industria en el sentido de no emplear o elaborar sustancias peligrosas.

Las exigencias ambientales dirigidas a productos repercuten asimismo en los métodos para fabricarlos.
Esto es importante, por cuanto las empresas que se ven obligadas a efectuar ajustes ambientales a corto plazo para ser competitivas no podrán eludir cambios estructurales de gran alcance a mediano y largo plazo. La evaluación ambiental de un producto suele basarse en el análisis de su ciclo de vida completo (UBA, 1992); incluye tanto los métodos de fabricación como las fases anteriores y posteriores a la producción (insumos, transporte, uso, eliminación). Así pues, para reducir el impacto ambiental de las propiedades del producto puede ser indispensable reestructurar la cadena entera de valor agregado, y no limitarse sólo a los necesarios ajustes de los métodos y procesos de producción. La política ambiental para el sector empresarial acentúa esa tendencia con una serie de incentivos para que las empresas asuman ellas mismas la gestión ambiental, incrementando su capacidad tecnológica y organizativa para resolver los problemas relacionados con ella. Forman parte de ese arsenal las normativas legales orientadas a procesos para prevenir cargas ambientales, instrumentos optativos como los balances ambientales y la participación en sistemas de gestión del ambiente y en auditorías ambientales. ${ }^{8}$ Esto no deja de repercutir en los proveedores de insumos: las empresas que mandan realizar auditorías o balances ambientales se fijan cada vez más en que sus subcontratistas cumplan con las exigencias ambientales en el proceso. Las empresas pioneras en cuestiones ambientales procuran la aptitud ambiental de un nuevo producto y de las distintas fases de su fabricación desde que empiezan a desarrollarlo, lo cual conlleva a su vez nuevas exigencias en lo tocante a redefinir la funcionalidad, la calidad y la durabilidad de los bienes.

\section{III}

\section{Presión en pro del ajuste ambiental y la competitividad sistêmica}

Mejorar la capacidad de ajuste ambiental en la empresa es un reto considerable a la capacidad de aprendizaje tecnológico y organizativo de las empresas exportadoras de los países en desarrollo. Con el agravante

\footnotetext{
${ }^{7}$ Bennett y Verhoeve (1994) ofrecen una visión detallada de las exigencias ambientales en la Unión Europea, Estados Unidos y Japón.
}

de que el contexto económico mundial y, por ende, los factores determinantes de la competitividad internacional han sufrido cambios estructurales, alcanzando un

\footnotetext{
${ }^{8}$ Karl (1993) y Hunt y Johnson (1993) ofrecen una reseña de la directiva de la Unión Europea sobre auditorías ambientales (véase Comisión Europea, 1993). Welford (1993) se ha referido a los límites de esos sistemas de manejo ambiental a nivel de empresa.
} 
Factores determinantes de la competitividad sistêmica

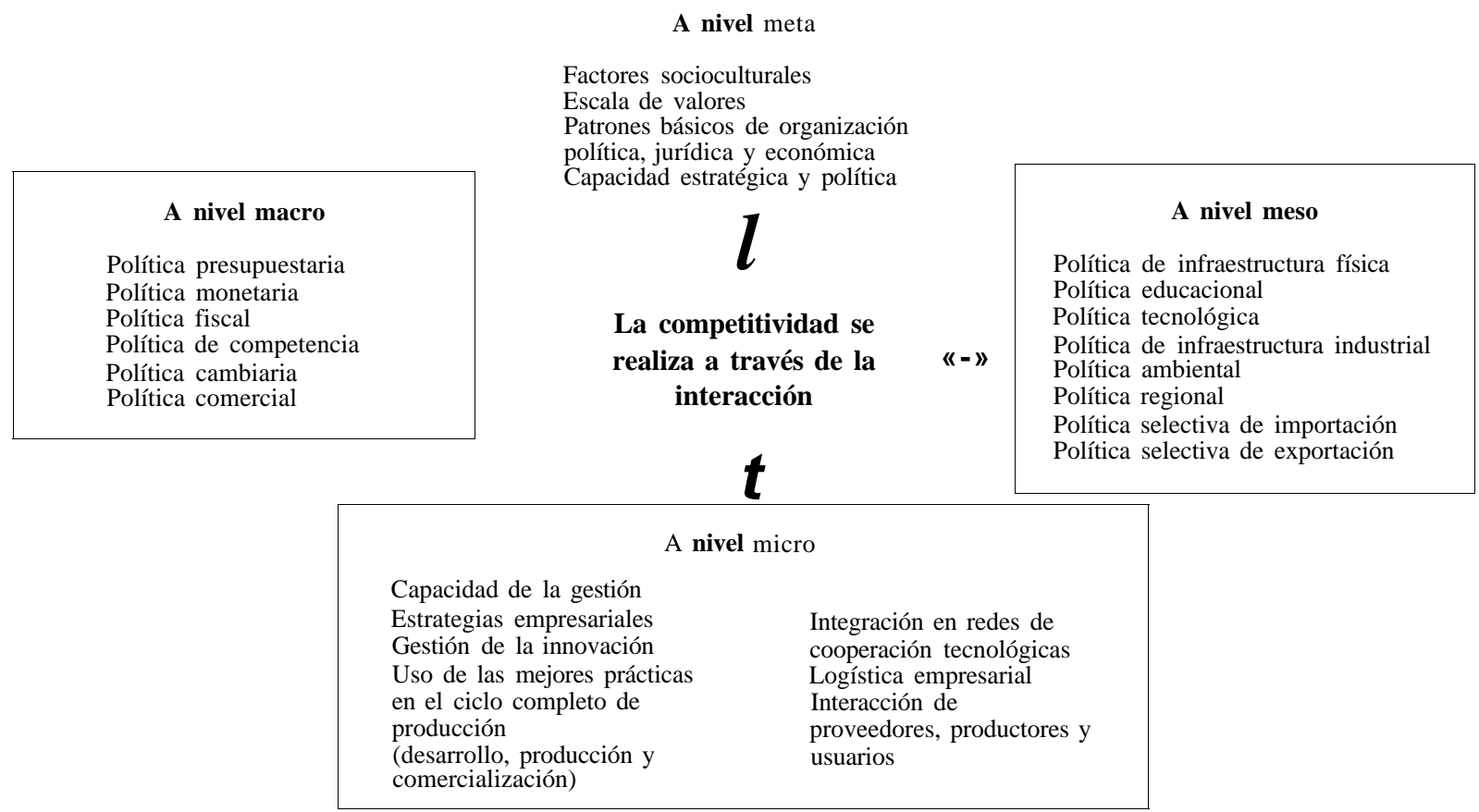

mayor grado de complejidad. El concepto de competitividad sistêmica (Esser, Hillebrand, Messner y Meyer-Stamer, 1994; Hurtienne y Messner, 1994) permite tanto diferenciar los planos en que deben emprenderse esfuerzos sistemáticos y articulados entre sí como identificar los grupos de agentes sociales involucrados (gráfico 1). Este concepto pone de relieve la urgente necesidad de crear estructuras concordadas y articuladas entre los distintos planos de acción y entre los grupos de agentes sociales.

Para mantener la competitividad internacional de la economía dentro de los límites de su sustentabilidad ecológica, los agentes tendrán que enfrentar el desafío de una mayor necesidad de interacción y coordinación entre los planos micro, meso y macro, generando nuevos instrumentos de gestión y un nuevo tejido institucional capaces de impulsar procesos de aprendizaje y de toma de decisiones dirigidos hacia un objetivo común. Queda claro por consiguiente que tanto los requerimientos ambientales de la competitividad como el modelo —algo esquemático por cierto- aquí formulado para conseguirla son de una exigencia extraordinaria, implican una serie de premisas y pueden plantear por lo tanto bastantes dificultades. En un contexto dominado por la competencia, el problema reside no sólo en cómo desarrollar estructuras concordadas y articuladas; las negociaciones que se han de entablar sobre los objetivos y prioridades del desarrollo y la forma de distribuir los beneficios esperados de la prosperidad económica entrañan un gran desafío en países cuyo débil tejido institucional dificulta la plena participación y la defensa de los intereses de todos lo\$ sectores de la población.

De modo análogo a las nuevas demandas que la conservación de la competitividad plantea a la capacidad de organización y aprendizaje de los agentes en los distintos planos de acción, la estrategia de desarrollo industrial ambientalmente sustentable hace indispensables complejos procesos de aprendizaje y ajuste en términos sociales, tecnológicos e institucionales. El nivel macro asume un papel significativo a la hora de crear las condiciones generales que permitan armonizar el desarrollo económico con el medio ambiente (legislación ambiental e instrumentos optativos y económicos). Los agentes estatales tienen que desarrollar los instrumentos ambientales necesarios y establecer las premisas legales para una política ambiental de cara al futuro, para sus objetivos y para las intervenciones necesarias del Estado, todo ello previo diálogo con los actores sociales (asociaciones empresariales, sindica- 
tos, instituciones científicas, organizaciones ambientalistas) para asegurar la legitimidad de estas medidas. Esta es una condición importante para movilizar el profesionalismo y la creatividad de las empresas y de los centros de investigación aplicada con miras a lograr soluciones prácticas y aptas para el medio ambiente. La aplicación de instrumentos económicos basados en el mecanismo de los precios para modificar comportamientos y mejorar la eficiencia también exige un amplio acuerdo entre los agentes sociales, políticos y económicos sobre la importancia de los objetivos que han de alcanzarse con tales medidas.

A nivel micro, lo que se busca es mejorar la disposición y la capacidad de las empresas para desarrollar estrategias activas tendientes a mejorar su eficiencia ambiental, que vayan más allá de los niveles mínimos de protección del ambiente y de los recursos naturales. Las empresas ecoeficientes trabajan para reducir el consumo de energía y recursos naturales de sus métodos de producción, para sustituir materiales o insumos contaminantes, para implantar tecnologías y productos limpios y para reciclar materias primas o productos (Schmidheiny y Consejo Empresarial para el Desarrollo Sostenible, 1992). ${ }^{9}$

La importancia del nivel meso se ve potenciada más aún por las exigencias que plantean las reformas estructurales de carácter ambiental a la capacidad de organización y aprendizaje de las instancias públicas y los agentes sociales. Esto concierne tanto a la elaboración de políticas sectoriales como al papel activo que pueden desempeñar las instituciones de este nivel en cuanto a crear y fortalecer la capacidad tecnológica. $^{10}$

Al diseñar políticas sectoriales y buscar solucio- nes específicas a nivel meso conviene concertar las medidas y programas ambientales con los sectores y grupos sociales involucrados para incrementar la legitimidad y el grado de aceptación, facilitando así la búsqueda de políticas y soluciones. Aunque decirlo parezca trivial, este paso es importante tanto para el legislador — cuya obligación es aprender a negociar con los grupos interesados pertinentes dentro y fuera del Parlamento y a justificar las intervenciones- como para las instancias públicas competentes. ${ }^{11}$ En este contexto sería razonable fomentar los esfuerzos descentralizadores con el fin de potenciar, a nivel regional o local, las capacidades para resolver problemas. Esto reviste particular importancia en los países en desarrollo, donde los órganos fiscalizadores no son lo bastante fuertes como para garantizar la eficacia práctica de los instrumentos legales y donde las estructuras democráticas son a menudo tan débiles que no permiten a las partes involucradas participar cabalmente en la elaboración de los necesarios instrumentos y medidas.

Fortalecer la capacidad tecnológica de las empresas es una condición clave para controlar mejor los impactos ambientales de los procesos de producción y de los productos. En consecuencia, las instituciones tecnológicas y el sistema educativo tienen que poder ofrecer el conocimiento técnico y los métodos y elementos necesarios a las empresas que quieren introducir innovaciones ambientales, por exigirlo así los mercados de exportación o el poder legislativo. Las instituciones que operan en la promoción de la pequeña y mediana industria, la transferencia de tecnología y el fomento a la exportación deben incluir de entrada la dimensión ambiental en sus actividades de asesoría y fomento.

\section{IV}

\section{La capacidad de ajuste ambiental de algunos sectores exportadores chilenos}

También los exportadores chilenos tienen que afrontar las exigencias ambientales que plantean los mer-

\footnotetext{
${ }^{9}$ Por su parte, North (1992) ofrece una visión general sobre las posibilidades de incorporar criterios ambientales a la gestión de empresas.

${ }^{10}$ Sobre la definición de tecnología y capacidad tecnológica, véase Hillebrand, Messner y Meyer-Stamer, 1994, pp. 3 - 8.
}

cados, sobre todo los europeos, a sus productos y métodos de producción. Para evaluar la capacidad de ajus-

\footnotetext{
${ }^{11}$ Los cinco años de atraso que lleva ya la aprobación de la Ley de Bosque Nativo en Chile ilustra lo difícil que puede resultar esta tarea en un contexto caracterizado, de un lado, por los márgenes recobrados de acción democrática y, de otro, por conceptos neoliberales y antiintervencionistas.
} 
te ambiental de los sectores examinados conviene clasificarlos en dos categorías: los sectores basados en recursos naturales, cuya competitividad internacional depende cada vez más de la capacidad de aplicar métodos de manejo sustentable, y los sectores manufactureros, cuya tarea consiste en mejorar la compatibilidad ambiental de sus métodos de producción y de sus productos para alcanzar niveles equiparables a los de los países industrializados.

\section{Sectores basados en recursos naturales}

Las empresas pertenecientes a estos sectores tienen una gran capacidad de ajuste. Las exigencias en materia de productos y procesos son relativamente abarcables y fáciles de satisfacer debido a su baja complejidad técnica.

\section{a) Cultivo de salmón, ostras y ostiones}

Respecto de este cultivo ${ }^{12}$ tanto la Unión Europea como Estados Unidos imponen normas rigurosas de higiene y salubridad a empresas y productos, así como de higiene a las aguas de cultivo. La observancia de tales exigencias debe ser supervisada y certificada por instancias oficiales reconocidas por las autoridades europeas o estadounidenses. La gran capacidad de ajuste de las empresas de este nuevo sector tiene su origen en cuatro factores claves: i) su creación se decidió desde un comienzo con miras a los mercados de exportación, de manera que su supervivencia ha dependido desde el primer momento de satisfacer exigencias externas; ii) las empresas tienen recursos para financiar el ajuste ambiental por ser solventes, por tener activos en reserva y por obtener cuantiosos beneficios gracias a sus altas tasas de crecimiento y a una acelerada expansión en los mercados del exterior; iii) las exigencias respecto a la calidad de las aguas pueden ser satisfechas sin esfuerzos previos por parte de las empresas, ya que Chile posee grandes superfi-

\footnotetext{
${ }^{12}$ Se efectuaron entrevistas a 10 empresas medianas y modernas, todas exportadoras y en su mayoría con participación extranjera. Las empresas de este sector se dividen casi siempre en dos secciones: el centro de cultivo y la planta procesadora. El procesamiento del salmón se limita a la cosecha, la limpieza, a veces el fileteado, el embalaje y la congelación o refrigeración; en el caso de las ostras y ostiones, al embalaje para su transporte refrigerado. La estructura relativamente homogénea de las empresas del sector confirió a la encuesta un carácter relativamente representativo. Fueron entrevistadas además todas las instituciones relevantes del nivel meso, entre ellas la Fundación Chile, el Servicio Nacional Pesquero (SERNAP), el Instituto de Fomento Pesquero (IFOP) y las asociaciones sectoriales.
}

cies de aguas interiores y costeras libres de contaminaciones de origen humano, y iv) en el mercado laboral, las empresas pueden satisfacer tanto la demanda de operarios bien preparados y especializados como la de mano de obra barata y no calificada.

Hay sin embargo un quinto factor clave de la elevada capacidad de ajuste en el nivel micro: que la estrategia elegida para desarrollar este sector implicó desde el principio la creación de un entorno institucional llamado a facilitar la cooperación entre las empresas del sector y las instituciones de investigación aplicada, información, transferencia de tecnología y control de calidad, con el fin de mejorar las posibilidades de supervivencia en los mercados de exportación. ${ }^{13}$ Esto quiere decir que dichas empresas han recogido desde su fundación experiencias positivas con formas de organización que les han permitido aprender conjuntamente y aunar esfuerzos para mejorar el nivel general de calidad y rendimiento del sector. ${ }^{14}$

Es de esperar en tal sentido que dicho sector será capaz de responder en la medida adecuada a una creciente presión (externa o interna, según el caso) para que se remedien los deterioros ambientales causados por la acuicultura, siempre que se trate de medidas a nivel de empresa. Esos deterioros se deben ante todo a la contaminación de las aguas por cultivos cada vez más intensivos y por los residuos de la cosecha, y comprenden además efectos negativos sobre la fauna local o regional. El sector, sin embargo, no podrá resolver los problemas causados por efluentes y otras fuentes de sustancias nocivas (como desagües comunales, agricultura, depósitos de basura) que pueden perjudicar su producción. Para conservar la pureza de las aguas de cultivo, las empresas estarán supeditadas a la emisión de normativas sectoriales, así como a la eficiencia de las autoridades regionales o locales que vigilan su cumplimiento y están en condiciones de coordinar el desenvolvimiento de los agentes involucrados en una región dada.

\section{b) Sectorforestal}

En el rubro de la madera, los importadores europeos inquieren con insistencia cada vez mayor por los métodos de manejo de los bosques o plantaciones que

\footnotetext{
${ }^{13}$ La Fundación Chile jugó un papel importante en este proceso (Schmidheiny y Consejo Empresarial para el Desarrollo Sostenible, 1992, p. 326 y ss.).

${ }^{14} \mathrm{La}$ asociación de la industria salmonera adjudica un sello de calidad que contempla todas las exigencias de los mercados de exportación y está reconocido por los importadores.
} 
dan origen a ese recurso natural. De ahí que el reto ambiental más grande que enfrenta el sector forestal chileno sea el de implantar métodos sustentables de manejo con miras a reducir el impacto ambiental de las plantaciones y a preservar el bosque nativo. ${ }^{15}$

El crecimiento del sector forestal empezó a acelerarse enormemente en 1974, una vez emitido el Decreto Ley 701, haciendo surgir en Chile las plantaciones de pino más extensas del mundo. ${ }^{16}$ La medida clave fue la de reprivatizar los bosques y ofrecer una garantía a la propiedad que abriera perspectivas seguras a una gestión forestal forzosamente orientada hacia los beneficios a largo plazo. Además, un $75 \%$ de los costos de reforestación y manejo forestal fueron objeto de subsidios y desgravaciones tributarias (Vincent y Binkley, 1992, p. 113). Esas medidas específicas de fomento han ayudado a convertir en fuentes de competitividad las ventajas comparativas naturales que posee Chile en este sector. ${ }^{17}$

Como consecuencia de esas medidas de fomento, el $90 \%$ de la producción forestal proviene de las plantaciones y sólo un $10 \%$ de bosques nativos. Las plantaciones se basan en una especie y están distribuidas sobre grandes superficies $(85 \%$ de pinos y el resto de eucaliptos), con una densidad generalmente muy alta. Producen los impactos ambientales típicos del monocultivo: consumo unilateral de nutrientes; reducción de la fertilidad del suelo con el peligro consiguiente de abono desmesurado; plantaciones altamente vulnerables a las plagas y las enfermedades, con los riesgos inherentes a la aplicación de pesticidas que con-

\footnotetext{
${ }^{15}$ Los entrevistados en este sector fueron las dos empresas más grandes en materia de plantaciones de pino insigne y eucalipto, empresas forestales de la XII Región (bosques de lenga), dos empresas productoras de astillas, así como pequeños propietarios de las zonas de bosques nativos de la IX Región. A nivel meso, fueron entrevistados en Santiago la Corporación Nacional Forestal (CONAF), el Instituto Forestal (INFOR), la Corporación Chilena de la Madera (CORMA), CODEFF — una organización no gubernamental- y expertos forestales de las principales universidades y de empresas consultoras; en la VIII, IX y XII Regiones, el grupo de trabajo visitó las oficinas regionales de manejo forestal con pequeños y medianos empresarios, así como los departamentos forestales de las universidades.

${ }^{16}$ Para frenar la deforestación del país, que ha venido avanzando con rapidez desde mediados del siglo XIX debido a la colonización del sur, las reforestaciones están desgravadas o subsidiadas desde 1931. En la actualidad hay plantadas más de un millón de hectáreas con Pinus Radiata, una conifera oriunda de Norteamérica. Según las estimaciones, esa superficie puede duplicarse o incluso triplicarse en el futuro.

${ }^{17}$ Entre las ventajas naturales de costos figuran un clima favorable y un suelo fértil -que mejoran enormemente el rendimiento de las plantaciones en comparación con el hemisferio norte-, así como los cortos trayectos hacia los puertos de exportación
}

taminan los recursos hídricos. Una contribución positiva de las plantaciones es que ayudan a combatir la erosión, ${ }^{18}$ que afecta por lo general a las superficies antes boscosas y luego desertizadas por la sobreexplotación agrícola. ${ }^{19}$

Las empresas grandes del sector han venido implantando modernos métodos de manejo forestal desde los años ochenta; esa modernización ha ayudado por una parte a mejorar la capacidad tecnológica y organizativa de las empresas, y por otra a crear las condiciones para que éstas perciban los problemas ambientales del sector y adapten poco a poco los métodos de manejo a los criterios ambientales. El primer paso por la senda de la modernización consistió en elevar la productividad estableciendo plantaciones de gran extensión, usando herbicidas y abonos sintéticos, mejorando las especies por medios genéticos y mejorando la calidad de la madera con métodos como la poda y el raleo. De esta manera, las empresas chilenas no tardaron en ponerse a la altura de las mejores prácticas internacionales en el sector forestal; por lo tanto, queda poco trecho hasta las innovaciones increméntales que vinculan los aumentos de productividad a la conservación de las funciones ambientales de los ecosistemas forestales.

Con el fin de mejorar la calidad de la madera, las empresas pioneras están concibiendo ahora las nuevas plantaciones con muy baja densidad y en forma de parques que permiten una biodiversidad considerablemente mayor; los herbicidas y los abonos se aplican con menor derroche y en forma adecuada a las condiciones locales; se ensayan métodos de control biológico de plagas; se opera con maquinaria de cosecha liviana que no deteriora tanto el suelo; la cosecha se realiza en superficies más pequeñas; se evita quemar los suelos después de la tala rasa para obtener más biomasa al dejar que se pudran las ramas y raíces, y la reforestación se emprende sin pérdida de tiempo para prevenir la erosión. Estos cambios tienen como trasfondo el hecho de que los métodos de manejo forestal

\footnotetext{
${ }^{18}$ El 70 a $80 \%$ de las plantaciones son reforestaciones en suelo degradado; el resto lo constituyen bosques nativos sustituidos ilegalmente. En contraste con los graves daños ecológicos que provocan las plantaciones de eucalipto en otras regiones del mundo, esta especie parece ser relativamente compatible con el medio ambiente chileno: en las plantaciones hay sotobosque, las abundantes precipitaciones son muy adecuadas al elevado consumo de agua de los árboles, y el eucalipto encuentra en Chile un habitat similar al de Australia, su país de origen.

${ }^{19}$ Sobre los daños por sobreexplotación agrícola y deforestación, sobre todo desde mediados del siglo XIX, véase Hajek, 1991.
} 
ambientalmente sustentable ejercen efectos positivos sobre los costos y ayudan a mejorar la calidad de la madera. Las medidas de ajuste se vieron facilitadas porque la estabilidad de los mercados y la rentabilidad de las plantaciones generaron los recursos financieros necesarios para invertir en investigación aplicada, mejorar los equipos y ensayar maquinaria nueva, y porque se contaba con ingenieros forestales bien preparados. Otro factor que impulsó la voluntad de ajuste a nivel micro fueron las empresas con participación internacional que dan importancia a determinados elementos de protección ambiental y seguridad laboral. $^{20}$

También las relaciones de cooperación entre las empresas (sobre todo en investigación aplicada), y entre éstas y los departamentos universitarios de ingeniería forestal, crean condiciones para asimilar con relativa facilidad el conocimiento técnico y organizativo que requiere el ajuste ambiental. Al interior del sector se dan procesos de aprendizaje imitativo por parte de los propietarios de plantaciones forestales pequeñas y medianas, que pueden comprobar el éxito de las innovaciones introducidas en los procesos por las empresas grandes; esta difusión la impulsan tanto las universidades que efectúan la investigación aplicada como las asociaciones empresariales.

En el sector forestal, el ajuste a nivel de empresa se ve perjudicado por la ausencia de una ley marco de alcance sectorial que regule la explotación de los recursos naturales (agua, suelo, bosque nativo) y por la falta de iniciativas regionales que muevan a cooperar a todos los actores involucrados para frenar el saqueo de esos recursos y reducir la contaminación de otros bienes ambientales (aquí cabrían las instancias fiscalizadoras y ambientales, los representantes comunales, los representantes de distintos sectores como el forestal, agrícola y turístico, las asociaciones ambientalistas y los representantes de los pequeños agricultores y de las comunas rurales). Es cierto que la Ley sobre Bases Generales del Medio Ambiente establece estructuras que deberían materializar esa coordinación regional - las comisiones regionales del medio ambiente (COREMA) cuentan con consejos consultivos in-

\footnotetext{
${ }^{20}$ Aquí se deja sentir la presión que ejerce la opinión pública en países con niveles ambientales y sociales más elevados: las agrupaciones ambientalistas controlan ahora las prácticas de las empresas transnacionales no sólo en su país de origen, sino además en otras localizaciones. Las empresas pertenecientes a los sectores que han ido a parar por razones ambientales al foco de la atención pública ya no pueden sacar ventajas comparativas trasladándose a países con niveles inferiores de protección del medio ambiente.
}

tegrados por representantes del sector privado, sindical y científico, así como de las agrupaciones ambientalistas-; pero también es cierto que la creación de esas estructuras tropieza con inconvenientes en la práctica, ya que - aparte del problema de la subfinanciación a nivel regional y local- el centralismo, un lastre del pasado, todavía dificulta movilizar en las distintas regiones los potenciales de organización y gestión política y social. Además, la capacidad de organización de los diferentes sectores sociales es heterogénea; es probable por lo tanto que estos consejos consultivos en muchos casos no alcancen un consenso equilibrado de intereses.

En lo que toca al manejo del bosque nativo, la falta de regulaciones sectoriales y de estructuras institucionales eficientes y bien dotadas (autoridades ambientales y forestales) es una de las causas fundamentales de la degradación progresiva de este bosque, ya que los intereses cortoplacistas de lucro y explotación logran imponerse con relativa libertad. ${ }^{21} \mathrm{La}$ implantación de métodos de manejo sustentable se ha visto inhibida por tres factores: i) la demora en aprobar el anteproyecto de Ley de Bosque Nativo (Jiles, 1992; Villarroel, 1992), que contempla la protección y el manejo adecuado de ese tipo de foresta; ii) la poca difusión de conocimientos sobre el manejo y el procesamiento eficiente y ambientalmente adecuado de las maderas nativas, y iii) el débil desarrollo de los mercados para los productos de maderas nativas con mayor valor agregado. Por estas razones, lo que predomina en las regiones pobladas de bosques nativos es la extracción de leña y la tala desordenada y destructora para obtener troncos destinados a la fabricación de astillas $^{22}$ y sustituir más tarde los bosques degradados por plantaciones. Ese tipo de explotación no es sustentable en términos forestales ni racional en términos económicos, ya que el valor agregado se mantiene bajo, el valor potencial de las maderas no se materializa y los efectos regionales o locales en cuanto a crear nue-

\footnotetext{
${ }^{21}$ No puede decirse que los intereses de lucro logren imponerse con entera libertad, por cuanto la Ley Forestal vigente ya exige que los propietarios de bosques presenten sus planes de manejo forestal a la CONAF para su aprobación. La capacidad de fiscalización de la CONAF es limitada; una advertencia importante consistió en las cuantiosas multas impuestas en 1994 a varias empresas del sudeste asiático por no cumplir lo establecido en los planes de manejo forestal chilenos.

${ }^{22}$ Las astillas son un insumo necesario para fabricar celulosa; Chile las exporta sobre todo a Japón. Los importadores japoneses piden astillas provenientes de maderas nativas para producir celulosa; de fibra corta - también lo hacen con astillas de eucalipto-, que e \$ la base para la fabricación de papeles de alta calidad.
} 
vos núcleos de crecimiento económico dinámico o nuevas fuentes de ingresos son muy precarios.

El potencial de desarrollo hasta ahora desaprovechado en la mayoría de los bosques nativos salta a la vista en comparación con el sector maderero del extremo sur chileno, que se ha desarrollado mediante un manejo cuidadoso de los bosques de lenga. ${ }^{23}$ Cuatro son las razones por las cuales se viene procurando desde hace algunos años manejar esa especie arbórea con criterios ambientales:

i) Las condiciones climáticas extremas impiden alcanzar los cortos ciclos de maduración que caracterizan a las plantaciones de pinos y eucaliptos de la VIII, IX y X Regiones. Por lo tanto, mantener plantaciones con especies exóticas no es más rentable que explotar la lenga, especie nativa que crece con lentitud, faltando así los incentivos para la sustitución.

ii) La lenga es una madera fina que sirve para fabricar partes y piezas de muebles destinadas a la venta en Estados Unidos y Europa. La madera de calidad inferior que se obtiene del raleo hecho para elevar la proporción de madera fina, es convertida en astillas y exportada al Japón, donde sirve para la producción de celulosa. Resulta, pues, que la fabricación de astillas ayuda a financiar el manejo forestal sustentable; el interés por obtener madera fina para la producción de piezas de muebles, que es más lucrativa, reduce el peligro de una tala masiva dirigida a la fabricación de astillas.

iii) La similitud de intereses de las empresas madereras que, obligadas a cooperar con vistas a satisfacer la demanda exterior, no practican una competencia perjudicial, y el hecho de que la industria maderera de esa región es muy joven y dinámica, han fomentado estrechas relaciones de cooperación dentro del sector, así como entre las empresas y los centros de investigación. Este sector, gracias a sus programas conjuntos de capacitación y perfeccionamiento, acusa en la actualidad un nivel de capacidad tecnológica relativamente elevado y homogéneo y una marcada voluntad de mejorarlo constantemente con miras a cumplir con las normas internacionales de calidad.

\footnotetext{
${ }^{23}$ Sobre el sector forestal o maderero de la XII Región faltan hasta ahora descripciones o análisis científicos; la única excepción es una evaluación de impacto ambiental que elaboró el Instituto Forestal (INFOR) con miras a un proyecto en Tierra del Fuego. El breve análisis aquí expuesto se basa en conversaciones sostenidas en la XII Región en marzo de 1994 con la CORMA Austral, la Trillium Corp., con expertos y consultores, con representantes de la CONAF y de la CORFO (fomento de la pequeña y mediana empresa) y con ProChile (fomento de la exportación).
}

iv) Estos esfuerzos se ven apoyados a nivel de empresa por un entorno institucional eficiente: existe una estrecha cooperación entre la CORMA Austral y la Universidad de Chile, un centro de excelencia para el desarrollo de métodos de manejo sustentable del bosque nativo, sobre todo de lenga. Junto con las oficinas regionales de la agencia estatal de fomento a la exportación (ProChile) y de fomento a la producción (CORFO), se están diseñando estrategias y programas para promover la industria maderera; forman parte de ellas las ideas para reducir el impacto ambiental, comercializar la madera y desarrollar la infraestructura física y la capacidad tecnológica de las empresas.

\section{Sectores manufactureros}

Las exigencias ambientales respecto a las propiedades del producto, así como la reconversión, el aumento de la eficacia y la racionalización de los procesos de producción en los sectores manufactureros son por regla general más complejos que en los sectores basados en los recursos naturales. Su observancia implica por lo tanto un elevado nivel de capacidad tecnológica, sobre todo en los sectores dedicados a la exportación. El entorno institucional de tipo tecnológico que rodea a los sectores industriales chilenos está poco desarrollado; faltan cursos de capacitación para obreros calificados; las instituciones existentes de investigación aplicada, de capacitación y de perfeccionamiento están poco articuladas entre sí. En los sectores analizados, estas debilidades estructurales del entorno repercuten de variadas formas en la capacidad innovadora de las empresas, según el grado de independencia (industrias globales en el sector de la celulosa) o dependencia (industrias con base nacional en el caso de la industria maderera) con que éstas puedan funcionar con respecto a su entorno.

\section{a) El sector de la celulosa}

El sector de la celulosa, orientado a la exportación, constituye un ejemplo de industria global enfrentada en todo el mundo con fuertes requisitos ambientales. De ahí la gran capacidad de ajuste que poseen las empresas del sector en términos de tecnología y organización. ${ }^{24}$ Como ocurre con la acuicultura, este

\footnotetext{
${ }^{24} \mathrm{Se}$ mantuvieron intensas conversaciones con cuatro empresas del sector y se visitaron dos plantas. A nivel meso se entrevistó a la CORMA - la asociación sectorial_, a la ATCP — la asociación de ingenieros-, a varios expertos independientes, a institutos y laboratorios universitarios de investigación, a ProChile y a la Fundación Chile.
} 
sector, cuya producción apunta sobre todo al mercado mundial, se ve obligado a perseguir los niveles internacionales de eficiencia, calidad y protección ambiental. La protección ambiental a nivel de empresa es en consecuencia muy elevada y en ciertos casos deja muy atrás las normativas vigentes en Chile. Las exigencias ambientales relativas a la celulosa apuntan sobre todo a los métodos usuales de blanqueo (sin cloro), la contaminación de los efluentes, el consumo de agua y energía y el manejo sustentable de las plantaciones de pinos y eucaliptos, de los que se extrae la madera para fabricar la celulosa. ${ }^{25}$

La gran capacidad de ajuste de las empresas se explica por la tecnología empleada (equipos, máquinas, técnicas, organización, conocimiento), que está estandarizada a nivel mundial. Habiendo suficiente capital disponible, es posible adquirirla con independencia del entorno local. Las empresas chilenas están integradas en redes tecnológicas internacionales en que se intercambia experiencia junto con conocimientos científicos y tecnológicos. Dentro de esas redes de cooperación se perfeccionan también los conceptos y métodos organizativos necesarios para aplicar el conocimiento y adaptar la técnica. ${ }^{26} \mathrm{El}$ intercambio informal de experiencia y de conocimientos prácticos sobre nuevas tecnologías con empresas e instituciones de investigación aplicada en el exterior se ve fortalecido en Chile por los institutos de investigación que igualan las mejores prácticas internacionales y cuyo financiamiento lo comparten las empresas nacionales de la celulosa; contribuyen al mismo objetivo los seminarios y talleres que organiza la Asociación Técnica de la Celulosa y el Papel (ATCP). Otro factor que facilita las cosas son las facultades de ingeniería chi-

\footnotetext{
${ }^{25}$ En respuesta a las campañas de información de las agrupaciones ambientales y a la sensibilización ya existente frente al reciclaje de papel (Greenpeace International, 1991 y 1993), la industria europea de la celulosa y el papel ha hecho ingentes esfuerzos para mejorar su perfil ambiental. Así, en los últimos años ha crecido enormemente la demanda de celulosa blanqueada sin cloro, elemental en el mercado europeo, que condiciona los suministros a pruebas detalladas de los bajos niveles de contaminación de la producción. Esta tendencia se está institucionalizando y fortaleciendo a través de los diferentes sellos ecológicos que Escandinávia y la Unión Europea dedican a los productos de papel. (Véase los requerimientos de sellos ecológicos para papel higiénico y rollos para cocina en Comisión Europea, 1994a y 1994b). El sello ecológico alemán se otorga sólo a los productos hechos íntegramente de papel reciclado.

${ }^{26}$ El sector de la celulosa se tornó interesante para la inversión extranjera en el curso de la privatización de empresas públicas y de la apertura al mercado mundial. Las tres nuevas plantas que iniciaron sus operaciones en 1991 y 1992 fueron financiadas por inversionistas multinacionales. Sobre la evolución del sector en Chile, véase Stumpo (1994) y DICELPA (1992).
}

lenas, de las que egresan profesionales muy bien preparados. Y a nivel de empresa, los cursos de capacitación y perfeccionamiento compensan las deficiencias técnicas de los operarios.

Resulta pues que, gracias a su buena situación financiera y a su integración a redes tecnológicas internacionales, las firmas productoras de celulosa están en condiciones de compensar los déficit estructurales del entorno institucional y asegurar su competitividad internacional. Pero, debido a su débil grado de articulación con las industrias nacionales y a su orientación exportadora, son pocos los impulsos de aprendizaje que genera este sector en la economía chilena en materia de protección ambiental a nivel industrial o empresarial. Visto así, las empresas del sector exportador de la celulosa pueden catalogarse de "islas de eficiencia ecológica" que no ejercen ningún papel precursor en pro de la protección del medio ambiente en el sector industrial chileno. Esa actitud tan cautelosa de las empresas concuerda más bien con la estrategia defensiva de la CORMA, asociación sectorial que rechaza las regulaciones con el argumento básico de que las intervenciones del Estado en el fuero particular son ilícitas. Extremando los términos, este sector podría considerarse como un ejemplo de modernización ambiental a medias, porque si bien es capaz de progresar tecnológicamente en aras de la competitividad, limita sus motivaciones a la lógica del mercado, de modo que sus efectos ambientales positivos son más que nada colaterales y asistemáticos.

\section{b) El sector de la madera}

El sector de la celulosa atraviesa por una situación distinta a la del sector de la madera, cuya capacidad actual de ajuste es limitada. ${ }^{27}$ Ello obedece a la complejidad de los requisitos y al grado de dificultad tecnológica, no pocas veces elevado, que implican las necesarias medidas de ajuste. El sector cuenta además con un alto porcentaje de pequeñas y medianas empresas (PYME) que padecen insuficiencias tanto financieras como de personal. Aquí se dejan sentir las difidencias del entorno tecnológico al servicio de las industrias, esto es, la falta de operarios expertos y bien adiestrados y la escasez de estructuras para la transferencia y la adaptación de nuevos métodos fabriles; pero

\footnotetext{
${ }^{27}$ Fueron entrevistadas doce empresas de diverso tamaño productoras de puertas, piezas de muebles y muebles, así como un fabri cante de pinturas, una empresa certificadora, doce instituciones del nivel meso dedicadas al fomento de las PYME, a la transferencia de tecnología y a capacitación y perfeccionamiento.
} 
además influyen las deficiencias estructurales generales, como la ausencia de una infraestructura pública para la eliminación de efluentes y residuos sólidos, o la imposibilidad de acceder a créditos para financiar las inversiones.

Las distintas exigencias ambientales apuntan actualmente a las sustancias tóxicas (prohibición del PCF, valores límite para las emisiones de formaldehído, nocividad de pinturas y lacas) y los materiales de embalaje; la sustentabilidad de los métodos de manejo forestal es un factor significativo para los mercados importadores europeos cuando se trata de productos de madera nativa. Si el nivel de protección ambiental en la industria europea del mueble no deja de crecer, el impacto ambiental de las técnicas de terminación (por ejemplo el laqueo) empleadas por los productores de terceros países que exportan a Europa, puede cortar el acceso a los mercados de ese continente. ${ }^{28}$

La capacidad de ajuste de las empresas del sector maderero acusa una gran diversidad por el carácter heterogéneo de éste. ${ }^{29}$ Dado que la competitividad de las empresas sigue siendo relativamente débil, la cuota de exportaciones de alta calidad a mercados sujetos a requisitos ambientales es todavía baja. En tal sentido no hay aliciente para atender a exigencias ambientales externas ni para emprender medidas de ajuste, ya que tales exigencias no existen en los segmentos de mercado que esas empresas abastecen, por ejemplo, en Estados Unidos. Pero a mediano y largo plazo, el desarrollo de ese sector dependerá de que las empresas incorporen elementos de protección ambiental a nivel tecnológico y organizativo en sus estrategias modernizadoras.

Otro obstáculo para que el sector tenga una mayor capacidad tecnológica y de respuesta a exigencias ambientales complejas son las débiles relaciones de

\footnotetext{
${ }^{28}$ Sobre los indicadores para medir y evaluar el impacto ambiental de la industria del mueble, véase Lehmann, 1993.

${ }^{29}$ Entre los aserraderos y la industria manufacturera, que generan más del $26 \%$ de la exportación de productos madereros, las empresas pequeñas, medianas y grandes aportaron un tercio de la producción total en cada caso. Las diferencias entre ellas en lo que se refiere a productividad y eficiencia son enormes. La industria de chapas y tableros se compone de 14 empresas medianas; la producción de puertas y molduras está en manos de ocho empresas pequeñas, 10 medianas y dos grandes; los dos subsectores aportan juntos alrededor del $4 \%$ de la exportación total. Sobre el número de plantas que operan en la industria del mueble no se dispone de datos concretos; en las tres categorías de tamaño se trata de un sinnúmero de fábricas que producen exclusivamente para el mercado interno o para la exportación, o que intentan colocar una parte de sus productos en el exterior. El desarrollo industrial del sector se ha caracte-
}

cooperación y coordinación entre las empresas. Un problema particular en este contexto es el suministro de insumos adecuados al medio ambiente, como madera aserrada sin tratamiento químico y pinturas y lacas ambientalmente inocuas.

Otros aspectos importantes en los que no influye todavía la protección ambiental son los controles de calidad y la certificación (inclusive de insumos y productos intermedios), la normalización, la asesoría tecnológica, la preparación de técnicos e ingenieros forestales, así como la investigación aplicada en materia de elaboración de la madera. Hay en el nivel meso una serie de instituciones que ya trabajan en dichas áreas, como el INFOR, en la certificación de la calidad de la madera, la Asociación de Industriales de la Madera (ASIMAD), en el control de la calidad y la certificación de productos finales, y el SERCOTEC y la CORFO en la asesoría tecnológica a empresas pequeñas y medianas. Pero son ante todo estas instituciones las que deberían mejorar su cooperación recíproca para impulsar los necesarios procesos de coordinación y concentrar esfuerzos. Esto encierra particular importancia para que los esfuerzos de investigación aplicada de las universidades beneficien a las PYME, cuyos limitados recursos no les permiten costear la investigación ni adquirir las soluciones técnicas disponibles.

El hecho de que los temas ambientales estén infravalorados en los planes de trabajo de las instituciones de investigación aplicada, asesoría tecnológica y capacitación profesional se debe a que las empresas y las instancias oficiales de protección ambiental generan poca demanda de soluciones de ese tipo. Sería conveniente entonces organizar medidas de perfeccionamiento y sensibilización para el personal de esos organismos, preparándolo así para las nuevas exigencias que van a recaer en las empresas.

rizado hasta ahora por la integración progresiva de las empresas forestales y los aserraderos. Sin embargo, esas empresas integradas abandonaron la producción de muebles de exportación tras algunos intentos que acarrearon fuertes pérdidas. Las más prósperas en materia de producción de muebles para exportar son algunas empresas medianas que ocupan entre 80 y 140 trabajadores, la mayoría de ellas concebidas desde un comienzo como fábricas de muebles que han encontrado nichos de mercado al cabo de intensas búsquedas. Más o menos el $50 \%$ de su producción encuentra cabida en los mercados europeos (Scholz, Block, Feil, Krause, Nakonz y Oberle, 1994, p. 58 y ss.). 


\section{V}

\section{Conclusiones y desafíos}

Con el propósito de sistematizar los factores que inhiben o promueven las medidas de ajuste ambiental en los sectores exportadores, los resultados del estudio en que se basa este artículo han sido clasificados por grupos de agentes sociales y por planos de acción dentro del concepto de competitividad sistêmica. Se intenta también explicar así algunos elementos de la interacción que el comercio mundial impulsa como una "correa de transmisión" entre los procesos de reforma ambiental en los países industrializados y los procesos de ajuste en los países en desarrollo.

\section{Nivel micro}

El punto de partida de la investigación fueron los procesos de ajuste ambiental en las empresas. El análisis de los casos exitosos (acuicultura, sector forestal, sector de la celulosa y sector del mueble en la XII Región) permite formular varios enunciados sobre los requisitos a nivel de la empresa que facilitan el ajuste frente a las exigencias relativas a productos y procesos. Son ellos los siguientes:

i) una estructura organizativa favorable a la innovación; en lo posible la existencia de un departamento ambiental que, en coordinación estrecha con las áreas de investigación aplicada, de personal y de ventas, desarrolle propuestas para mejorar los procesos de producción y los productos (en su gama de ofertas y propiedades), recogiendo las sugerencias innovadoras de los trabajadores;

ii) una gestión empresarial consciente de la necesidad de innovar continuamente en los procesos de producción y de elevar la eficiencia;

iii) la existencia de profesionales bien preparados (ingenieros) que tengan el conocimiento necesario para implantar innovaciones ambientales;

iv) la capacitación y el perfeccionamiento constantes del personal, sobre todo de los obreros calificados y los técnicos;

v) la articulación en redes empresariales a nivel sectorial (o nacional o internacional, según el caso) de actividades conjuntas en las áreas de investigación aplicada, capacitación, ventas y comercialización;

vi) la creación de un instrumental capaz de asimilar estándares externos de calidad y eficiencia y de impulsar los pasos de ajuste apropiados en el marco de la orientación exportadora general de las empresas.

\section{Nivel meso}

Un entorno empresarial eficiente es un requisito básico para apoyar y facilitar las medidas de ajuste que se tomen a nivel de la empresa a medida que en su gestión se van incorporando los aspectos ambientales (instituciones de orientación tecnológica, sector educativo). Las organizaciones intermedias (asociaciones empresariales, instituciones sectoriales de fomento y asesoría) pueden desempeñar al mismo tiempo una importante función de bisagra entre los niveles macro y micro con vistas a concertar objetivos ambientales, crear los instrumentos para avanzar hacia ellos y mejorar las posibilidades de alcanzarlos. En los cases analizados se ha podido comprobar que, para efectuar debidamente el ajuste a nivel de empresa, es preciso que en el entorno institucional se den los requisitos siguientes:

i) instituciones tecnológicas que investiguen con fines de aplicación práctica y ofrezcan servicios adecuados a las empresas dispuestas a la innovación, para que ensanchen sus áreas de trabajo con un componente ambiental y asimilen conocimiento específico. Esto abarca, en el sector forestal, el manejo sustentable de las plantaciones de pino y eucalipto, el control biológico de plagas, el mejoramiento genético de las espécies, y el manejo de los bosques nativos; en la industria maderera, el desarrollo de métodos no contaminantes de conservación y tratamiento de la mader^, incluida la nativa y en el sector de la celulosa, métodos de blanqueo sin cloro, ensayo y certificación de calidades de la pulpa, y la medición, ensayo y certificación del gasto ambiental de origen industrial;

ii) seminarios, talleres y grupos de trabajo en las asociaciones sectoriales que faciliten un aprendizaje imitativo y permitan que las empresas tengan un funcionamiento homogéneo en términos de calidad y protección ambiental (acuicultura, sector forestal, industria del mueble en la XII Región);

iii) planes de estudio de las instituciones de enseñanza profesional y cursos de capacitación adaptados a los nuevos requerimientos empresariales; 
iv) posibilidad de un estrecho engranaje de las actividades de las asociaciones sectoriales, así como de las instituciones de investigación aplicada y de capacitación y perfeccionamiento. ${ }^{30}$

Si el entorno empresarial está en condiciones de ejecutar estas tareas, serán grandes las posibilidades de responder adecuadamente a las exigencias externas -incluso si éstas son complejas y tecnológicamente intensivas-, y de evitar que las empresas se vean sobreexigidas y desplazadas del mercado.

Las empresas grandes integradas en redes de cooperación internacional pueden compensar las insuficiencias propias de un entorno empresarial débilmente estructurado y mal articulado, manteniendo en alto su nivel de competitividad (sector de la celulosa). En cambio, las empresas pequeñas y medianas dependen para su ajuste ambiental de los servicios que les brinde su entorno (manejo forestal, industria del mueble); lo que necesitan no es sólo ayuda tecnológica, sino además infraestructura física (por ejemplo, sistemas comunales de tratamiento y eliminación de residuos y efluentes industriales).

\section{Nivel macro}

En el contexto que incentiva las medidas de ajuste ambiental en las empresas cabe señalar dos factores esenciales:

i) Si la actividad está condicionada por la existencia de recursos naturales, este hecho puede impedir que se sobreexplote estos recursos si es que no hay manera satisfactoria de sustituirlos (industria del mueble basada en la madera de lenga);

ii) la orientación del modelo económico hacia la competitividad y el mercado mundial obliga a las empresas a tomar medidas permanentes para incrementar la competitividad y la eficiencia o para regirse por los estándares internacionales de calidad y eficiencia; como las mejores prácticas tecnológicas son por regla general más eficientes que las tecnologías más antiguas en términos de consumo de energía y materias primas, la asimilación de aquéllas surte por lo

\footnotetext{
${ }^{30}$ Los dos últimos requisitos todavía no se han hecho realidad en Chile, pero los entrevistados los calificaron de deficiencias. Al presentar los resultados obtenidos, los representantes de los institutos de capacitación y perfeccionamiento revelaron un vivo interés por esa temática; durante las discusiones, todas las instituciones del nivel meso (de investigación aplicada, de transferencia de tecnología, de fomento a las PYME, e instancias sectoriales) enfatizaron la necesidad de coordinar y articular mejor sus actividades.
}

menos efectos colaterales positivos para el medio ambiente (sector de la celulosa, sector forestal).

De no existir una política ambiental independiente que señale objetivos de ajuste y protección a nivel sectorial, las empresas insistirán en mantener una estrategia defensiva y de reacción en lugar de movilizar y optimizar su potencial para resolver los problemas que las afectan. Para los nuevos sectores exportadores dependientes de los recursos naturales, esto sugiere que las regulaciones ambientales promotoras de un manejo más eficiente de esos recursos pueden acelerar el paso de la explotación extensiva de materias primas a la intensiva, con el consiguiente desarrollo de sectores manufactureros de mayor valor agregado (la Ley Forestal persigue explícitamente ese doble objetivo). ${ }^{31}$ Por lo tanto, una política ambiental activa puede contribuir a la creación de nuevas ventajas competitivas.

\section{Nivel internacional}

Transmitidas a través del comercio con países cuyas normas ambientales son más rigurosas, las exigencias ambientales legales o del mercado sobre los productos o procesos o sobre las fases de preproducción y posproducción pueden desencadenar e impulsar procesos de aprendizaje e innovación tecnológicos y organizativos en pro del medio ambiente. Un requisito importante para potenciar esa interacción son los mercados abiertos: las empresas exportadoras respetan las normativas ambientales cuando éstas son implantadas en los mercados importadores. Sin embargo, la disposición de las empresas a invertir en la modernización con fines ecológicos se debilita cuando hay razones para dudar de la seriedad de las exigencias ambientales (proteccionismo encubierto) y cuando los mercados importadores potenciales resultan casi inaccesibles para productos más sofisticados desde el punto de vista de la protección ambiental. Las normativas proteccionistas, si van en aumento, pueden anular los efectos positivos de los requisitos ambientales impuestos a productores de terceros países, induciéndolos a desviar su comercio hacia países que no plantean tantas exigencias con respecto a los productos.

Los efectos contraproducentes de las normas proteccionistas sobre la disposición de las empresas de

\footnotetext{
${ }^{31}$ La explotación intensiva, en contraste con la extensiva, se basa en los principios de la eficiencia (no derrochar recursos naturales) y de la sustentabilidad (explotar recursos naturales respetando su ciclo de regeneración natural y conservar las funciones del ecosistema al que pertenece cada recurso natural).
} 
terceros países no es el único argumento contra las medidas comerciales de orden ambiental que varias asociaciones empresariales y sectores industriales de los países industrializados demandan en el debate a favor del "Greening ofGatt". Una política de comercio con orientación ambiental también tiene que contemplar las condiciones iniciales específicas —económicas, tecnológicas, organizativas y ambientales-en que produce la empresa. Por ejemplo, si unos agentes sociales débiles se ven obligados a encarar exigencias que sobrepasan a corto plazo su capacidad de innovación y ajuste, el resultado puede ser la desviación de la exportación hacia mercados menos rigurosos en sus requisitos ambientales. Los procesos de política am- biental pueden verse retrasados si las exigencias ambientales se identifican con los intereses proteccionistas, impidiendo ver los problemas reales subyacentes.

La comprobación de que el comercio internacional puede impulsar procesos de aprendizaje ambiental en países exportadores en desarrollo como Chile pone de relieve otro hecho importante: el avance de las reformas ambientales en los países industrializados puede hacer que también en los países en desarrollo se abran los necesarios márgenes políticos y económicos para implantar patrones de producción más adecuados al medio ambiente.

(Traducido del alemán)

\section{Bibliografía}

Bennett, G. y B. Verhoeve (1994): Environmental Product Standards in Western Europe, the US and Japan. A Guidebook, Arnhem, Países Bajos, Instituut voor Europees Milieubeleid.

(bfai) Bundesstelle fur Aussenhandelsinformationen (1995): Landerreport Chile. Wirtschaftstrends zum Jahreswechsel 1994/95, bfai Landerreport, Colonia, bfai, 1995

Chile (1994): Ley sobre bases generales del medio ambiente, Diario Oficial de la República de Chile, $\mathrm{N}^{\circ} 34.835$, Santiago de Chile, 9 de abril.

CIPMA (Centro de Investigación y Planificación del Medio Ambiente)(1992): Gestión ambiental en Chile, Aportes del $4^{\circ}$ Encuentro científico sobre el medio ambiente, Santiago de Chile.

Comisión Europea (1993): Directiva del Consejo sobre la participación voluntaria en un sistema comunitario de gestión ambiental y de auditoría ambiental (1836/93), Boletín de las Comunidades Europeas, L 168, Bruselas, Comunidades Europeas, junio.

(1994a): Resolución de la Comisión del 14 de noviembre de 1994 para fijar criterios ambientales para la concesión del sello ecológico de la CEE para papel higiénico (94/924/CE), Boletín de la Unión Europea, $\mathrm{N}^{\circ} 364 / 24$, Bruselas, Comunidades Europeas, diciembre.

(1994b): Resolución de la Comisión del 14 de noviembre de 1994 para fijar criterios ambientales para la concesión del sello ecológico de la CEE para papel de cocina (94/925/CE), Boletín de la Unión Europea, $\mathrm{N}^{\circ} 364 / 32$, Bruselas, Comunidades Europeas, diciembre.

DICELPA (Federación Técnica Iberoamericana de la Celulosa y el Papel) (1992): Directorio de la industria de la celulosa forestal y del papel: productores y proveedores 1992-1993, Santiago de Chile, Organización PuntoDiez.

Dockendorff, E. y otros (1990): Chile en sus regiones. Materiales para el análisis y la acción, Santiago de Chile, Centro de Estudios del Desarrollo (CED).

Esser, K., W. Hillebrand, D. Messner y J. Meyer-Stamer (1994): Competitividad sistêmica. Competitividad internacional de las empresas y políticas requeridas, Berlín, Instituto Alemán de Desarrollo (IAD)

Gómez-Lobo, A. y J. Jiles (1993): La experiencia chilena en regulación pesquera, O. Muñoz (éd.), Después de las privatizaciones: hacia el Estado regulador, Santiago de Chile, Corporación de Investigaciones Económicas para Latinoamérica (CIEPLAN).
Greenpeace International (ed.) (1991): Weniger Müll, mehr Wald. Konzepte zum Einsparen und Recyceln von Papier, Hamburgo. (1993): Papier - Naturprodukt oder Chemiecocktail, Hamburgo.

Hajek, E.R. (1991): Chile, la situación ambiental en América Latina. Algunos estudios de casos, Buenos Aires, Centro Interdisciplinario de Estudios sobre el Desarrollo Latinoamericano (CIEDLA)

Hillebrand, W., D. Messner, y J. Meyer-Stamer (1994): Fortalecimiento de la capacidad tecnológica en países en desarrollo. Enseñanzas de la cooperación técnica alemana, Berlín, IAD, diciembre.

Hunt, D. y C. Johnson (1993): The systems approach to corporate environmental management and environmental auditing, Business Strategy \& Environment, vol. 2, № 1, Shipley, Reino Unido, ERP Environment.

Hurtienne, T. y D. Messner (1994): Nuevos conceptos de la competitividad, Cambio de rumbo en el Cono Sur. Los casos de Argentina, Chile y Uruguay, Caracas, Editorial Nueva Sociedad.

Jha, V., R. Vossenaar y S. Zarrilli (1993): Ecolabelling and International Trade, Ginebra, Conferencia de las Naciones Unidas sobre Comercio y Desarrollo (UNCTAD).

Jiles, J. (1992): Análisis económico: valoración social vs. valoración privada, dilema clave para una regulación efectiva del bosque nativo, Ambiente y Desarrollo, Santiago de Chile, Centro de Investigación y Planificación del Medio Ambiente (CIPMA).

Karl, H. (1993): Europâische Initiative für die Einfiihrung von Umweltschutz-Audits; Kritische Wiirdigung aus okonomischer Sicht, List-Forum, vol. 19, $\mathrm{N}^{\circ} 3$, Baden-Baden, Alemania, Nomos.

Lehmann, S. (ed.)(1993): Umwelt-Controlling in der Mbbelindustne; Ein Leitfaden, Berlin, Institut für õkologische Wirtschaftsforschung.

Madrid, R. y C. Ominami (1989): Lincamientos estratégicos para una inserción activa en los mercados internacionales, Santiago de Chile, Instituto Latinoamericano de Estudios Internacionales (ILET).

Maggi, C. (1994): Descentralización territorial y competitividad: El caso de Chile, Berlín, IAD.

Mármora, L. y D. Messner (1992): Chile im lateinamerikanischen Kontext - Ein Modell für Demokratisierung und Entwicklung in der gesamten Region?, J. Ensignia y D. Noite (éd.), Mo- 
dellfall Chile? Ein Jahr nach dem demokratischen Neuanfang, vol. 34, Hamburgo, Instituto de Estudios Iberoamericanos.

Messner, D. (1993): Búsqueda de competitividad en la industria maderera chilena, Revista de la CEPAL, $\mathrm{N}^{\circ}$ 49, LC/G. 1757-P, Santiago de Chile 1993, CEPAL.

North, K. (1992): Environmental Business Management; an Introduction, Ginebra, Organización Internacional del Trabajo (OIT).

OCDE (Organización de Cooperación y Desarrollo Económicos) (1991): Environmental Labelling in OECD Countries, París.

Sanhueza, G. (1992): Der Umweltschutz: eine weitere Herausforderung an die Demokratie, en J. Ensignia y D. Noite (eds.), Modellfall Chile? Ein Jahr nach dem demokratischen Neuanfang, vol. 34, Hamburgo, Instituto de Estudios Iberoamericanos.

Schmidheiny, S. y Consejo Empresarial para el Desarrollo Sostenible (1992): Cambiando el rumbo. Una perspectiva global del empresariado para el desarrollo y el medio ambiente, México, D.F., Fondo de Cultura Económica (FCE).

Scholz, I. (1993): Requerimientos ambientales a productos de consumo en Alemania y sus efectos sobre las exportaciones de los países en desarrollo, Berlín, IAD.
Scholz, I., K. Block, K. Feil, M. Krause, K. Nakonz y C. Oberle (1994): Medio ambiente y competitividad: El caso del sector exportador chileno, Berlín, IAD.

Stumpo, G. (1994): Desempeño, competitividad y perspectivas del sector de papel y celulosa en Chile, Santiago de Chile, CEPAL.

UBA (Oficina Federal del Medio Ambiente) (1992): Òkobilanzenfur Produkte, Berlín.

Villarroel, P. (1992): ¿La ley que todos esperaban?, Ambiente y Desarrollo, vol. 8, $\mathrm{N}^{\circ} 3$, Santiago de Chile, Centro de Investigación y Planificación del Medio Ambiente (CIPMA).

Vincent, J.R. y C.S. Binkley (1992): Forest-based industrialization: A dynamic perspective, en N.P. Sharma (ed.), Managing the World's Forests. Looking for a Balance Between Conservation and Development, Washington, D.C., Banco Mundial.

Wiemann, J. y otros (1994): Ecological Product Standards and Requirements as a New Challenge for Developing Countries' Industries and Exports. The Case of India's Leather, Textile and Refrigeration Industries, Berlin, IAD.

Welford, R. (1993): Breaking the link between quality and the environment: Auditing for sustainability and life cycle assessment, Business Strategy \& Environment, vol. 2, $\mathrm{N}^{\circ} 4$ Shipley, Reino Unido, ERP Environment. 